Rev. Psicol. (Arequipa. Univ. Catól. San Pablo) / Año 2019 / Vol 9 / № 3 / pp. 95-103

ISSN 2306-0565 versión impresa / ISSN 2311-7397 versión on line

(c) $\left(\begin{array}{l}(1) \\ \text { Esta obra está bajo una Licencia Creative Commons } \\ \text { Atribución 4.o Internacional (BY-NC-ND) }\end{array}\right.$

\title{
CONSTRUCCIÓN DEL SENTIDO DESDE LAS NARRATIVAS DE LOS MIEMBROS DE UNA ORGANIZACIÓN
}

\section{CONSTRUCTION OF IN MEANING FROM NARRATIVES OF MEMBERS OF AN ORGANIZATION}

\author{
Eduardo Escalante', Ignacio Fernández y Pía Cordero ${ }^{2}$ \\ 1. Universidad Juan Agustín Maza, Mendoza, Argentina \\ 2. LEAD Institute, Santiago, Chile
}

\begin{abstract}
Resumen
En este artículo, se describe la construcción de significado en las prácticas organizativas utilizando el paradigma de la construcción naturalista del sentido creado por Kurtz y Snowden (2003) como una forma de examinar los acontecimientos de una organización lejos de los procesos naturales de control y la opinión de expertos. Las narrativas, las historias, las interacciones son elementos fundamentales de las acciones colectivas culturales en una organización. De este modo, sus acontecimientos se tratan más allá de un enfoque positivista. Se postula una metodología para construir el sentido (narrative sensemaking). Este método sugiere escuchar las diferentes historias de los diferentes actores de la organización y clasificarlas. Díadas o tríadas se utilizan términos distintivos, que permiten a los actores contar sus historias y sus percepciones en un marco cualitativo y cuantitativo en lo posible, lo que depende de herramientas computacionales. Este proceso permite a una combinación única de un análisis estadístico (objetividad de los números) con el contexto rico proporcionada por la evidencia anecdótica. Una vez recogidas las distintas narrativas, se organiza un taller de interpretación. En esta etapa, los consultores ayudan a encontrar patrones de captura que emerge del número de señales de historias. Las señales débiles pueden ayudar a anticipar las tendencias futuras y ayudar a tomar decisiones sobre futuras estrategias e intervenciones.
\end{abstract}

Palabras clave: Complejidad, construcción de sentido, narrativas, historias. 


\begin{abstract}
This paper describes the construction of meaning in organizational practices using the naturalistic construction of meaning paradigm created by Kurtz and Snowden (2003) as a method of examining organizational events apart from the natural processes of control and expert opinion. Narratives, the stories and interactions, are fundamental elements of the cultural collective actions of an organization. Thus, organizational events are addressed beyond a positivist approach. A methodology for constructing meaning is proposed (narrative sensemaking). This method suggests listening to the different stories of the different actors in the organization and classifying them. Dyads or triads employ distinctive terms, allowing actors to tell their stories and describe their perceptions in a qualitative framework, and where possible a quantitative framework, depending on the computational tools to be used. This process allows a unique combination of statistical rigor (objectivity of numbers) along with the rich context provided by anecdotal evidence. Once the various narratives are collected, an interpretation workshop is organized. At this stage, consultants help to find emerging patterns captured from the number of story cues. Weak cues can help to anticipate future trends and help make decisions about future strategies and interventions.
\end{abstract}

Key words: Complexity, sensemaking, narratives, stories.

\section{Introducción}

Hace un tiempo venimos trabajando los conceptos de Kurtz y Snowden (2003), con respecto al análisis de la complejidad y el uso de las narrativas, que permiten analizar determinados procesos en una organización. Específicamente, los conceptos de historias y significantes. El término historia no denota algo bien construido y ejecutado, sino que representa la experiencia cruda vivida por un individuo, que correspondería a un GPS orientador para traducir la complejidad en gestión efectiva y hacerlo resiliente (propiedad emergente de los sistemas adaptativos complejos).

Nuestra existencia transcurre en un mundo enteramente delimitado. En éste, establecemos tanto límites naturales como artificiales. Al usar el concepto de "límite" se alude de uno u otro modo a una discontinuidad. Y eso ocurre en las organizaciones. En toda organización hay historias de fracasos y éxitos, nudos gordianos sin resolver, límites que superar. Es decir, siempre se está trazando líneas o límites para organizar su experiencia propia o ajena. Los límites ordenan la acción y le confieren sentido. Pero, así como hay una actitud delimitadora hay una actitud desdelimitadora: remover los límites. Más aún si nos movemos en dominios que no están ordenados y que son complejos. Las distinciones que hemos hecho no funcionan, no son efectivas, $y$, por ende, es preciso examinarlas y posiblemente modificarlas. Es por ello que se habla de la fenomenología 
del límite que nos ilumina también en los casos en que se extralimita, donde no se trata de suprimir un límite, sino de traspasarlo. El tema es indagar que impulsa a hacerlo o no hacerlo.

Ungran aportede la construcción de sentido narrativo en relación con las metodologías convencionales es la desintermediación. Esto significa que los expertos intervienen solamente para mediar el proceso de construcción de sentido, permitiendo que los miembros de la organización clasifiquen sus historias según categorías, o etiquetas quellamamos significantes. Se pueden usar díadas o tríadas de términos distintivos y también se puede partir de un problema o de datos estadísticos resultantes de la aplicación de una escala de medición.

\section{Modelo y metodología de análisis}

Antes de continuar con este proceso metodológico. Describiremos el modelo de análisis de la complejidad de Snowden (1999): dominios simple, complicado, complejo, y caótico. Este autor habla de la multiontología de la construcción del sentido. Es una especie de cartografía que es en realidad una "ontología epistemológica", porque lo que busca es hacer identificaciones y distinciones: orden/ desorden; conocido/desconocido. Y todo ello para poder definir lo que se podría ver como la antropología que ocurre en cada escenario o dominio; e indagar en el sentido de las distintas actuaciones reales y posibles o simplemente imposibles. Este autor explora lo que nos distingue y define en cada dominio (orden/desorden), agregaríamos lo que nos incumbe, nos importa, nos preocupa. En este proceso se nos va el sery el de los otros con los que interactuamos. Es decir, el transcurrir de la existencia humana y las diversas identidades que van surgiendo en cada dominio y en los límites de éstos. Un enfoque de esta índole permite darse cuenta que la identidad se va transformando con el tiempo y que por lo mismo no es sino una manifestación del ser de la plenitud, del ser que se individúa y fenomeniza en la organización, entre otros. El autor ofrece así, un conjunto de distinciones que permiten aproximarse a la gestión de sistemas humanos.

Snowden identifica cuatro escenarios, dominios o contextos para la toma de decisiones: conocido (simple), conocible (complicado), complejo y caótico. Los dos primeros corresponden a sistemas ordenados, en los que los patrones que se visualizan tienden a repetirse con pocos cambios. Las causas conducen a efectos confiables y observables y no se esperan mayores sorpresas. La diferencia entre el primeroy el segundo es lo que actualmente se conoce y lo que se puede hallar.

Los dominios complejo y caótico caen en el territorio de los sistemas sin orden, esto es, sus patrones no son predecibles, incluso si existe mucha información. Los patrones en un espacio complejo dependen de las interacciones entre entidades, incluyendo observadores. Los patrones complejos no se repiten, lo que hace difícil predecirlos. Sin embargo, hay patrones que aparecen y desaparecen. La diferencia entre la complejidad y el caos deriva del hecho que la primera genera patrones relativamente simples de una variedad grande de 
interacciones, en cambio el segundogenera patrones intrincados. Representa un orden de otro tipo: el no orden.

Hoy las organizaciones enfrentan complicaciones y complejidades del entorno y de sí mismas (las interacciones de los actores), el orden simple no es la tendencia necesariamente, hay demasiados elementos emergentes, cada uno generando sus principios, lógica y conocimiento. Esto hace interesante las elaboraciones de Snowden (2002) para hacer explícitos los supuestos de las personas y/o grupos ante un tema particular. En la figura 1, se puede observar que la dinámica organizacional, funciona con un orden determinado (si hubiera desorden, simplemente dejaría de existir). Como hemos señalado más arriba, en este orden, hay cuatro escenarios posibles: simple, complicado, complejo, y caótico. Cada tema de la organización se puede representar en algunos de estos espacios de la matriz, además de la intensidad (tamaño del círculo) y orientación que tengan (positiva/negativa).

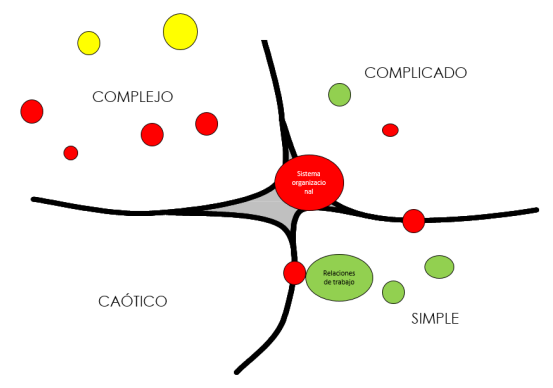

\section{Figura 1. Dominios de compeljidad}

La experiencia indica que una persona puede tener interpretaciones múltiples sobre un tópico en particular basado en el contexto en el que se experimenta ese tópico. Es el poder performativo de las palabras. Comprender que tenemos identidades múltiples es tan importante como comprender que tenemos realidades múltiples. Nuestras identidades no tienen supuestos que son siempre consistentes. Por ejemplo, uno puede ver el tópico de la disciplina de manera muy diferente como padre que como empleado de una organización. Además, las identidades múltiples requieren que se tome en cuenta la naturaleza del contexto histórico y local.

El esquema de la figura 1 no intenta proveer una visión fija de la realidad sino más bien un pantallazo en el tiempo que se puede comparar con otros pantallazos para explorar patrones y trazar movimientos. La utilidad de éste radica en la habilidad para apoyar a los individuos y/o grupos en la toma de decisiones que requieren tomar en cuenta diversos puntos de vista ante un determinado fenómeno. El foco se centra en la toma de decisiones (Kurtz \& Snowden, 2003). Esta noción de utilidad es consistente como lo que se denominada "construcción social de la realidad".

Tradicionalmente los enfoques positivistas para hacer indagaciones operan bajo el supuesto que las cosas existen en el mundo y pueden ser descubiertas y que podemos aprender sobre ella y comprender su naturaleza "verdadera". Es común preguntarse sobre el "rigor" de la información cuando se examina una investigación desde la perspectiva positivista. Esto es, se busca la validez del estudio. Pero, ¿rigor con respecto a qué? ¿en qué se basa? 
Se asume que el conocimiento es fijo y tiene propiedades indiferentemente de nuestra comprensión de esas propiedades. Si cambiamos nuestra orientación desde un mundo que existe fuera de nosotros a una orientación donde el mundo es una combinación de interacciones, en constante co-evolución el mundo mismo, entonces se puede traspasar muchos de los obstáculos filosóficos paradójicos en el campo de las ciencias sociales. Se produce un desplazamiento y el valor de la información y el conocimiento ya no está en el rigor y validez, sino en su utilidad en un determinado momento local histórico. Además, el valor en la utilidad no es un valor fijo en el tiempo, dado que va emergiendo nueva información.

Desde nuestro punto de vista, se puede argumentar que la figura anterior se puede utilizar para maximizar la utilidad proveyendo a los participantes la oportunidad para externalizar sus supuestos acerca de un tópico particular. Esto permite lograr una comprensión compartida sobre un tema y se constituye en el primer paso en la consideración de las soluciones o toma de decisiones.

Se trabaja con el sentido como un suceso, una acción, pero también con el sentido en cuanto justificación (del suceso, de la acción), y finalmente con el sentido en tanto orientación, lo queda dirección. Además, se pueden descubrir los "focos del sin-sentido", esto es, fenómenos, sucesos, situaciones que revelarían pérdida de sentido: molestia, fastidio, cansancio, etc. Se puede detectar las fuentes ocasionales y persistentes del sentido (que revelarían un determinado patrón o tendencia).

Seusa el término "comprensión compartida" en contraste con "consenso". Esto porque la meta no necesariamente es el alcanzar el consenso, puede ocurrir que a la espera del consenso se esté instalando el estatus-quo. Se denota que los participantes estén concientes de los puntos de vista múltiples ante un tema en particular incluso si no están de acuerdo.

Al final del día, la estructura de poder en el lugar puede tomar la decisión final, pero de esta manera la decisión se tomará a la luz de todos los participantes. Si hay un desequilibrio se destacará en este proceso. De este modo, la toma de decisiones se entenderá en un nivel más profundoy será más difícil descartara aquellos que podrían ser impactados negativamente. El consenso denota que todos los involucrados están de acuerdo con una decisión particular. Se sustenta en la noción de una verdad o solución particular.

Lo anterior permite convocar a la escena a Cynthia Kurtz que ofrece una variedad de métodos que incorporan la recopilación de información desde perspectivas múltiples. Kurtzy Snowden (2003) desarrollado herramientas computacionales para realizar el proceso. El primero ofrece SenseMaker que es muy apropiada para este tipo de análisis, pero bastante costosa; la segunda propone Narrafirma, herramienta también bastante poderosa que es gratuita.

Las historias narradas por las personas son filtros a través de los que 
toman decisiones (Snowden, 2005). Usan material fragmentado para dar sentido al mundo alrededor de ellas. Metodológicamente, la combinación de datos duros (estadísticos) con datos blandos (narrativas) se pueden construir en sentido cuando se abordan realidades complejas, en orden a destilar patrones y responder de manera oportuna. En el caso de las organizaciones, revela el mundo a través de los ojos de sus actores (Oliver \& Snowden, 2005).

El punto de partida puede ser una pregunta (por ejemplo, cuáles son las creencias sobre algo que no funciona), una imagen, una situación, un dato estadístico (un promedio de las respuestas ante un reactivo). Estos elementos desencadenan una "experiencia vivida" que le da significado al acontecer, lo importante es que ahora se recurre a la interpretación dada por otros (al diálogo apreciativo). Esto permite detectar patrones en las historias, discutirlos y construir conglomerados (clusters), para finalmente, actuar sobre esos patrones. Se pueden simular patrones beneficiosos y desechar los no deseables. Se puede usar una tríada (tres aspectos de un dominio de interés). El uso de formas triangulares permite la tensión creada entre tres puntos equidistantes como alternativas; los participantes se ven forzados a considerar detenidamente su elección reduciendo el prejuicio de la conformidad (esto es, la tendencia a seleccionar lo mejor, fácil o la respuesta esperada en una escala mono-dimensional.

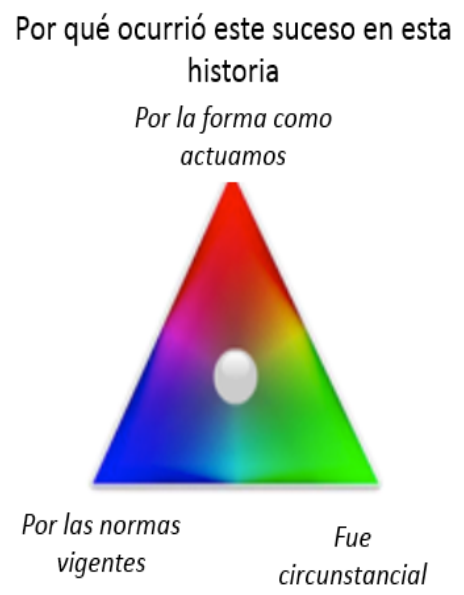

\section{Figura 2. Ejemplo 1 de Tríada}

Algunas tríadas pueden permitir observar la diversidad de la ética humana y determinar el sentido del locus moral de la responsabilidad. Como un dominio amplio, que involucra temas de conciencia, obligación, deber, culpa y la estructura de la experiencia moral. La tríada no focaliza lo que debería ser, sino que pone el énfasis en lo que es. La tríada de la responsabilidad moral enfatiza lo personal más que lo social (ver figura 2).

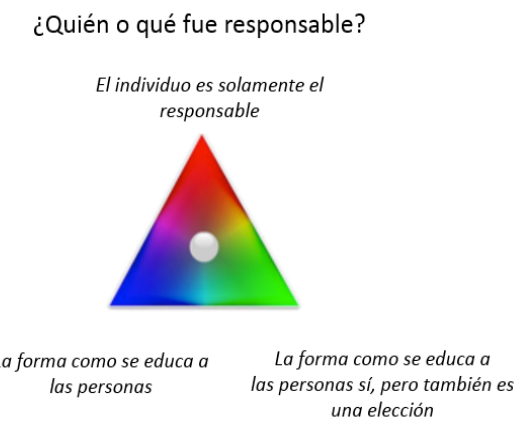

Figura 3. Ejemplo 2 de Tríada 


\section{(2) Observador/es}

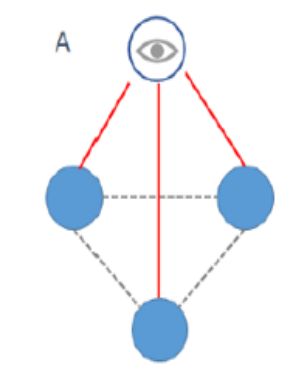

Conexiones centrales fuertes Interacciones distribuidas débiles

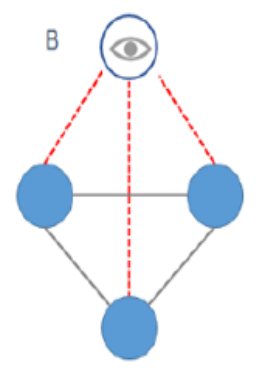

Débil conexiones centrales Interacciones distribuidas débiles

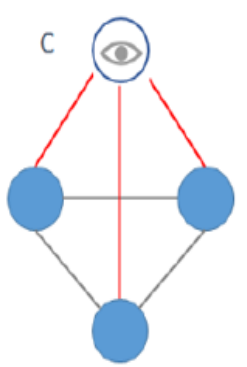

Conexión

débil

Conexión

fuerte

\section{Figura 4. Tipos de conexiones}

Los actores de la organización (observadores) podrán identificar a través de sus narrativas los vínculos que existen en un determinado momento entre sus diferentes estructuras. Aquí, es preciso aclarar que en este enfoque quien trabaja con las historias no dice o interpreta o cambia o selecciona las historias, nunca. Todos estos procesos los realizan solamente las personas en un grupo de interés. La mediación permite pensar, hablar, compartir e identificar patrones, pero se respeta la integridad de la historia como materia prima en bruto.

Se trata de la identidad colectiva de la resiliencia de una organización, o su habilidad para gestionar las múltiples identidades sin quiebres (mantener la suficiente diversidad de aquellas identidades) que es poyada por la identidad de resiliencia de sus empleados.

El análisis de datos cuantitativos y cualitativos de una historia y las formas de significación revelan un número de tendencias o temas que son representados en la matriz de la figura 1. Algunos temas residen en un dominio específico. Otros se posicionan en los límites de dos escenarios entre dominios adyacentes para indicar la presencia contemporánea de características típicas de diferentes dominios (por ejemplo, un tema muestra características de un sistema complicado y complejo). El color del tema puede indicar positivo, neutral, o negativo. Para cada tema el tamaño del círculo es proporcional a la recurrencia de este tema en las historias (esto es, temas que aparecen en muchas historias se representan en círculos más grandes que los temas que aparecen en pocas historias). Cuando un tema es muy recurrentey la solución se ve compleja, entonces se requiere que un experto loaborde usando sus conocimientos y capacidades. En especial, cuando hay más de una solución posible y solamente se puede optar después de una cuidadosa evaluación de los diferentes criterios. 


\section{Conclusiones}

La ontología epistemológica de diferentes dominios propuesta por Snowden (1999): dominio simple, complicado, completo y caótico, es un ordenamiento interesanteque permite a su vez identificar comportamientos antropológicos en las organizaciones a través del estudio de las narrativas de sus actores. Este tipo de enfoque permite que los sistemas complejos de una organización estén alertas a las señales de formación de patrones. La cognición está distribuida de manera diferente según ciertos umbrales numéricos: las organizaciones trabajan con 50 menos individuos, 15 o menos, 150 o menos. Snowden describequince como el "límite de confianza" y cinco como el límite de la memoria de corto plazo.

La investigación de las historias y el compaginarlas en la matriz de Snowden, permitiría construir el sentido en las organizaciones empleando procesos mixtos: cuantitativos y cualitativos. De este modo, muchos temas que no se visualizan con métodos tradicionales podrán emerger con toda la riqueza de su poder descriptivo. Los temas que caigan en el dominio simple serán de fácil solución, más trabajo se requerirá en el dominio complicado y las narrativas de los actores permitirán con el conocimiento experto identificar soluciones a partir de los patrones identificados y analizados. Si se trata de un tema que cae en el borde o dentro del dominio complejo, se requerirá experimentación como vía para aprender y comprender sus patrones, indudablemente se requerirá paciencia para lograr una solución práctica y aceptable. Aplicar un enfoque de decisiones impropias puede ocasionar efectos contraproducentes. 


\section{Referencias}

Kurtz, C., \& D. Snowden. (2003). The New Dynamics of Strategy: sense making in a complex complicated world. IBM Systems Journal 42(3), 462-483.

Oliver, G. R., \& Snowden, D. J. (2005). Patterns of narrative in organizational knowledge sharing: Refolding the envelope of art-Luddism and techno-fabulism. En G. Schreyögg, \& J. Koch (Eds.), Knowledge management and narratives: organizational effectiveness through storytelling (pp. 15-72). Berlin: Erich Schmidt Verlag.

Snowden, D. J. (1999). The Paradox of Story: Simplicity and Complexity in Strategy, Scenario and Strategy Planning, 1(5), 16-20.

Snowden, D. J. (2002). Complex acts of knowing: Paradox and descriptive self awareness. Journal of Knowledge Management, 6(2), 100-111.

Snowden, D. (2005). Stories from the Frontier. E:CO Issue, 7(304), 155-165.

Snowden, D. J., \& Boone, M. E. (2007). A Leader's Framework for Decision Making. Harvard Business Review, November, 69-76.

Recibido: 25 de agosto de 2017

Modificado: 14 de octubre de 2018

Aceptado: 19 de marzo de 2019 